ORIGINAL ARTICLE

\title{
Shoulder pathoanatomy in marathon kayakers
}

\author{
G Hagemann, A M Rijke, M Mars
}

Br J Sports Med 2004;38:413-417. doi: 10.1136/bjsm.2002.003699

See end of article for authors' affiliations

.....................

Correspondence to: Dr Rijke, University of Virginia, MSE-SEAS, 203 Thorntol Hall, MSE-SEAS, Charlottesville, Virginia 22903, USA; amr@virginia.edu

Accepted 20 May 2003

\begin{abstract}
Objectives: To determine the prevalence of soft and hard tissue abnormalities and their interrelations in the shoulders of marathon kayakers and to examine the pathoanatomical factors that predispose these athletes to injury.

Methods: Fifty two long distance kayakers completed a questionnaire. Their shoulders were examined for range of motion, pain, and stability using a standard set of 10 clinical tests. The shoulder was subsequently scanned by magnetic resonance imaging (MRI) in three planes and evaluated for evidence of injury or other abnormality. The relation of clinical symptoms and MRI findings was investigated with respect to kayaker's age, number of years kayaking, and number of marathon races completed.

Results: Thirty subjects were asymptomatic at the time of scanning, and twenty two showed symptoms of pain and/or instability. MRI showed acromioclavicular hypertrophy, acromial or clavicular spur, supraspinatus tendinitis, and partial tear of the supraspinatus as the most common abnormalities. Kayaker's age, number of years kayaking, and number of races completed did not relate significantly to symptoms or to the presence of an abnormality on MRI scan. Of all the pathoanatomical findings that are reported to predispose to rotator cuff injury, only acromial and clavicular spurs were found to correlate highly with supraspinatus muscle pathology.

Conclusions: Rotator cuff injuries make up a large portion of the injuries seen in marathon kayakers, about twice the number reported for sprint kayakers. These injuries are the result of secondary impingement factors associated with overuse, possibly specific to kayakers, and not the result of bony restrictions around the shoulder joint. Acromioclavicular hypertrophy is a common finding in marathon kayakers, but is possibly the result of portaging or a previous injury.
\end{abstract}

Very little work has been carried out specifically on shoulder injuries in kayaking. Walsh ${ }^{7}$ stated that shoulder injuries can be benign and self limiting, yet occasionally can cause cessation of hard paddling, an opinion shared by Cox and Nouwen after collating the results from a questionnaire on injuries in sprint kayakers. These authors found that out of the 30 international participants, 16 reported shoulder injury, which broke down into 14\% shoulder bursitis, $20 \%$ biceps tendinitis, and $20 \%$ rotator cuff tears with the remainder showing no notable lesions. ${ }^{6}$

An understanding of shoulder injuries in kayakers is best obtained from an appreciation of the kinesiological, biomechanical, and physiological demands of the sport. The rotator cuff muscles provide the forces to generate movement in the shoulder and are intimately involved in stabilising and controlling the humeral head in the glenoid during the kayak stroke. The effectiveness of the cuff depends on its force of action, which is related to its size, type, and speed of contraction. It is also related to its moment arm or leverage and to its angle of pull.

At the beginning of the paddling cycle, the attacking, leading shoulder is stretched forward and the corresponding arm is extended and horizontal. The opposite (active) shoulder is moved backward behind the head in abduction, extension, and external rotation. In the first phase, the traction arm remains extended while the torso rotates and the legs push against the footbar, thus transmitting the thrust to the paddle. In the second phase, the traction arm flexes until the forearm reaches a minimum of $90^{\circ}$. As the blade passes the hip of the kayaker, the opposite shoulder adducts with internal rotation and the corresponding arm is actively pushed forward. Throughout these phases, the likelihood of mechanical irritation within the shoulder complex is increased. Passage through the water is made with a rapid

three types were identified: ro
bursitis, and/or biceps tendinitis. 
outward rotation of the traction arm after which the return phase begins.

In clinical practice, overuse injuries to the shoulders of sportsmen are more common than traumatic injuries or pathology after surgery. According to Zuluaga et $a l^{8}$ the anatomy and biomechanics of the shoulder girdle, combined with the demands placed on it in the pursuit of sporting excellence, would predispose the shoulder to this type of injury.

Inflammation of the rotator cuff appears to be the main problem affecting kayakers' shoulders. ${ }^{5}$ However, the reason why certain people develop rotator cuff inflammation while others do not is far from clear.

According to Neer, ${ }^{10}$ the vast majority of such cases are due to primary impingement of the rotator cuff muscles/ tendons as a result of the anatomical restrictions of the subacromial space. The contents of this narrowed space would rub against elements of the coracoacromial arch when repetitive shoulder action is performed, especially in elevation and internal rotation, which eventually leads to compressive tendinitis. The structure exposed to maximum impingement is the area of insertion of the supraspinatus tendon, but the biceps tendon, the subacromial bursa, and even the acromioclavicular joint may be involved as well. ${ }^{11}$ Others, such as Nirschl, ${ }^{12}{ }^{13}$ disagree. They argue that the primary cause is multiple repetitions of stretch injury under contractile load, or intrinsic overload of the musculotendinous unit, leading to tensile tendinitis. It is true that impingement does complicate the process, but it is due to swelling of the subacromial bursa and/or inflammation of the muscle/tendon and is thus secondary in nature. Secondary impingement may also result from pain, which causes reflex inhibition and weakness of the rotator cuff muscles which, in turn, fail in their function to centre the humeral head in the glenoid. Subsequent translation superiorly then adds to the impingement by further decreasing subacromial space. Other factors such as poor scapular control, capsular laxity, and abnormal biomechanics may also contribute to secondary impingement. ${ }^{14-16}$

To determine the prevalence of soft and hard tissue abnormalities and their interrelations in the shoulders of paddlers, a clinical study of experienced kayakers was undertaken and the results compared with the findings of magnetic resonance imaging (MRI). Anatomical factors that possibly contributed to predisposing these athletes to injury were examined, and the pathogenesis of any of these injuries was evaluated.

\section{PATIENTS, METHODS, AND MATERIALS}

A total of 52 kayakers were recruited from volunteers who met the following criteria for eligibility:

- Participation in kayaking events for seven or more years

- Completion of at least one endurance race a year with a minimum of seven races (where an endurance race is defined as any race longer than $120 \mathrm{~km}$ ).

Exclusion criteria were: last endurance race more than two years ago, previous surgery to shoulder to be scanned, and the general contraindications to MRI scanning such as implanted metal clips and claustrophobia.

The kayakers ranged in age from 27 to 58 with a mean of 40.2 years. Two of them were women. They had been participating or competing in the sport for a mean of 17.2 years (range 8-35). Individually, they had completed on average 19.6 endurance races (range 7-53), each over a distance of more than $120 \mathrm{~km}$ (total of $104 \mathrm{l}$ endurance races).
The study was performed with the approval of the bioethics committee of the University of Natal. All subjects were first interviewed and gave written informed consent. Next, they were asked to complete the questionnaire. Their shoulders were then examined by the same practitioner (GH) using a standardised approach.

Both shoulders of the subjects were assessed for active range of motion-that is, flexion/extension, abduction/ adduction, internal/external rotation, and horizontal flexion. Ten clinical tests were selected to determine the integrity of the structures associated with the shoulder girdle, taking into account the sensitivity and specificity, complexity of test, time constraints, and appropriateness.

- Anterior apprehension test, to test for anterior instability of the glenohumeral joint. ${ }^{8}$

- Posterior apprehension test, to test for posterior instability of this joint. ${ }^{17}$

- Inferior sulcus sign, to assess laxity in an inferior direction. ${ }^{8} 18$

- Resisted internal rotation, to test the integrity of the subscapularis muscle. ${ }^{17} 19$

- Resisted external rotation, to assess infraspinatus and teres minor muscles. ${ }^{17} 19$

- Centinela test, which isolates the supraspinatus in a tensile test. ${ }^{20}$

- Speed's test, also named Yergason's test, is a tensile test for bicipital tendinitis. ${ }^{17} 21$

- Impingement tests. These tests attempt to compromise the suprahumeral soft tissues by compressing them against a portion of the coracoacromial arch. They are diagnostically non-specific and do not identify the structure responsible for the pain on impingement. The test according to Neer and Welsh, cited in Falkel and Murphy, ${ }^{17}$ involves a forced flexion of the humerus in internal rotation, jamming the subacromial structures against the anterior third of the acromion. The one according to Hawkins and Kennedy ${ }^{11}$ has both shoulder and elbow flexed with the humerus internally rotated to drive the greater tuberosity beneath the coracoacromial arch.

- Acromioclavicular joint compression, to assess acromioclavicular joint pathology. ${ }^{8}$

Active range of motion and the above 10 tests were scored on a four point scale.

MRI scans were performed using a GE Signa $1.5 \mathrm{~T}$ whole body scanner. A wrap around surface coil designed for the shoulder was used throughout. The shoulder was scanned with the subject supine and the arm in anatomical position. Routinely, the choice of shoulder to be scanned was alternated with respect to the previous patient, unless a particular shoulder had been symptomatic by the patient's own account, in which case that shoulder was chosen. However, if a shoulder had had previous surgery, the opposite shoulder was scanned. Patients were prevented from falling asleep during the scanning procedure to avoid heavy breathing movement artefacts.

Images were acquired in the axial, coronal oblique (parallel to the long axis of the belly of the supraspinatus muscle), and the sagittal oblique (perpendicular to the coronal oblique) planes.

Fast spin echo (FSE) sequences were used throughout. Tl weighted (TR 700 milliseconds, TE 15 milliseconds) images were collected for all 52 patients and T2 weighted (TR 4000 milliseconds, TE 96 milliseconds) images were collected for 39 patients. However, none of the 13 patients who did not have a $\mathrm{T} 2$ weighted scan showed any abnormality on their $\mathrm{Tl}$ weighted images. For imaging, a $256 \times 256$ matrix was used. 
Pixel size was $0.75 \mathrm{~mm}^{2}$, and the field of view (FOV) was $16 \mathrm{~cm}$. Image slice thickness was kept at $4.0 \mathrm{~mm}$, with a $1.0 \mathrm{~mm}$ gap.

All scans were read by the same radiologist (AMR), who was blinded to the results of the questionnaire and physical examinations. The following anatomical structures were systematically evaluated in each case.

- The subacromial bursa was analysed for signal intensity, thickness, and anatomical course.

- The rotator cuff tendons were studied for signal intensity and anatomical course.

- The rotator cuff muscles were analysed for signal intensity and their integrity.

- Spurs from the anteroinferior margin of the acromion, from the acromioclavicular joint or clavicle were searched for.

- Hypertrophy of the acromioclavicular joint was assessed. This could be due to trauma with or without optimal healing, or have osteoarthritic origins or both.

- Hypertrophy of the subscapularis and supraspinatus muscles was estimated by measuring their diameters at the widest point in the belly.

- The morphology of the coracoid process, specifically any lateralisation, was determined.

- The acromion was evaluated for its appearance in the different planes and for the presence of an os acromiale. The normal appearance of an acromion on MRI is either a flat undersurface or a concave undersurface congruent to the curvature of the upper aspect of the humeral head, best appreciated in the coronal and sagittal planes. Lateral downsloping shows as angulation in the aspect of the undersurface on coronal views. Anterior downsloping shows on sagittal views.

- The coracohumeral distance was measured as the shortest distance including the cartilage between the respective bony anatomy on axial scans. Lateral extension of the coracoid process with decreased coracohumeral interval can be the cause of coracoid impingement of the subscapularis muscle/tendon.

- The acromiohumeral distance was measured on the coronal oblique images to assess the space available to its contents, specifically the supraspinatus muscle/tendon.

- The acromial arch and the coracoacromial ligament were searched for their integrity and normalcy.

\begin{tabular}{|c|c|c|}
\hline Symptom description & $\begin{array}{l}\text { Number of } \\
\text { subjects }\end{array}$ & $\begin{array}{l}\text { MRI } \\
\text { positive }\end{array}$ \\
\hline $\begin{array}{l}\text { (1) Asymptomatic at the } \\
\text { time of PE/scanning }\end{array}$ & $30(58)$ & $13(43)$ \\
\hline (2) Asymptomatic always & $23(44)$ & $10(43)$ \\
\hline $\begin{array}{l}\text { (3) Asymptomatic at time of PE/ } \\
\text { scanning but symptomatic (pain } \\
\text { only) previously }\end{array}$ & $7(13)$ & $3(43)$ \\
\hline $\begin{array}{l}\text { (4) Symptomatic at the } \\
\text { time of } \mathrm{PE} / \text { scanning }\end{array}$ & $22(42)$ & $14(64)$ \\
\hline Pain only & $13(25)$ & $8(61)$ \\
\hline Instability only & $4(8)$ & $2(50)$ \\
\hline Pain and instability & $5(10)$ & $4(80)$ \\
\hline (5) Symptomatic (pain) always & $11(21)$ & $8(73)$ \\
\hline $\begin{array}{l}\text { (6) Symptomatic at time of PE/ } \\
\text { scanning but asymptomatic }\end{array}$ & $8(15)^{\circ}$ & $5(62)$ \\
\hline
\end{tabular}

Values in parentheses are percentages. MRI, Magnetic resonance imaging; PE, physical examination.

\begin{tabular}{|ll|}
\hline $\begin{array}{l}\text { Table } 2 \text { Summary of magnetic resonance } \\
\text { imaging findings }\end{array}$ \\
\hline Finding & $\begin{array}{l}\text { No of } \\
\text { subjects }\end{array}$ \\
\hline Acromioclavicular joint hypertrophy & 15 \\
Acromial or clavicular spur & 11 \\
Supraspinatus tendinitis & 6 \\
Partial tear of supraspinatus & 4 \\
Fluid in sheath of long head of biceps & 1 \\
Subscapularis tendinitis & 1 \\
Partial tear of subscapularis & 1 \\
Subacromial bursitis & 2 \\
Coracoacromial ligament rupture & 1 \\
Os acromiale & 1 \\
Ganglion cyst & 1 \\
No pathology & 25 \\
\hline
\end{tabular}

\section{RESULTS}

\section{Symptomatology}

The results of the physical examination of the 52 subjects were grouped on the basis of pain, instability, and time of occurrence. They are applicable to the scanned shoulder only and summarised in table 1 .

In addition, five kayakers remembered a particular incident causing a sudden onset of pain. However, it was difficult to ascertain whether these injuries were acute, acute on chronic, or an aggravation of a chronic injury. Five subjects had required surgery to a shoulder, in which case the opposite shoulder was selected for scanning.

\section{MRI findings}

Table 2 gives a summary of the pathology detected on MRI scans; 27 of the 52 scans showed an abnormality (52\%).

Tables 1 and 2 show that about half of the patients were permanently symptom-free and about half also showed no abnormality on MRI, but the patients in these two groups were not necessarily the same. However, this distribution of symptoms and MRI findings within the study group permits the valid application of statistical tests to analyse the data.

The patients' symptoms were recorded as pain, instability, or pain with instability derived from any of the 10 clinical tests and the active range of motion assessment. Of the previous symptoms, only pain could be determined reliably. MRI findings were classified as any abnormality or specific abnormalities. As shown in table 2, the most common MRI abnormalities were acromioclavicular joint hypertrophy and acromial/clavicular spurs with their presumed associated subacromial bursa, supraspinatus muscle/tendon, and biceps tendon involvement.

Mean age, number of years kayaking, and number of endurance races completed were considered in relation to symptoms and MRI findings (table 3).

Table 3 Results of Mann-Whitney tests relating symptomatology and magnetic resonance imaging (MRI) findings with respect to age, years kayaking, and number of races completed

\begin{tabular}{|c|c|c|c|}
\hline & $\begin{array}{l}\text { Symptomatic at } \\
\text { time of scan }\end{array}$ & $\begin{array}{l}\text { Symptomatic at } \\
\text { any time }\end{array}$ & MRI findings \\
\hline Age & 0.342 & 0.433 & 0.161 \\
\hline $\begin{array}{l}\text { Number of years } \\
\text { kayaking }\end{array}$ & 0.198 & 0.122 & 0.323 \\
\hline $\begin{array}{l}\text { Number of races } \\
\text { completed }\end{array}$ & 0.123 & 0.867 & 0.331 \\
\hline
\end{tabular}


Table 4 Asymptomatic subjects with shoulder pathology on magnetic resonance imaging

\begin{tabular}{ll}
\hline Pathology & No of subjects \\
\hline Acromioclavicular joint hypertrophy & 5 \\
Acromioclavicular spur & 3 \\
Old tear of supraspinatus & 1 \\
Old tear of subscapularis & 1 \\
Os acromiale, acromioclavicular spur, & 1 \\
supraspinatus tendinitis & \\
\hline
\end{tabular}

Eleven kayakers claimed never to have experienced any symptoms but showed pathology on MRI nonetheless. Table 4 lists their MRI findings.

In principle, the data from tables 1-4 allow the determination of the role of impingement within the framework of injured shoulders of kayakers. To this end, shoulder symptoms were correlated with respect to specific anatomical abnormalities as seen on MRI. The presence of the following abnormalities were investigated: (a) an acromial or clavicular spur; $(b)$ lateral or anterior downsloping of the acromion; $(c)$ an os acromiale; $(d)$ acromioclavicular hypertrophy. One or more of these pathoanatomical variables were tested against whether the kayaker was (a) currently asymptomatic but had experienced symptoms previously (seven subjects), (b) always asymptomatic (23 subjects), (c) currently symptomatic but previously asymptomatic (eight subjects), or (d) symptomatic both currently and in the past (11 subjects). However, using Fisher's exact test, no statistical significance was found for any of the above variables.

The above mentioned four pathoanatomical variables were also correlated with the presence or absence of a tendinitis or tear, either current or past, in one of the rotator cuff muscles/ tendons. Owing to the small sample of pathoanatomical variables and the number of subjects with rotator cuff pathology, only the presence or absence of an acromial or clavicular spur was compared. Fisher's exact test showed that there was a significant difference between these two variables (numbers are listed in table 5).

Other pathoanatomical variables such as the supraspinatus diameter, acromiohumeral distance, and their ratio, as well as the subscapularis diameter, coracohumeral distance, and their ratio were investigated with respect to the presence or absence of symptoms both for those kayakers who were only currently symptomatic and for those who were either currently and/or previously symptomatic. No significant difference was found between groups for any of these variables. Furthermore, no significant difference was found between the acromiohumeral distance and MRI evidence of supraspinatus tendinitis $(\mathrm{p}=0.062)$, although this does approach significance. There were not enough positive cases to investigate coracohumeral distance with respect to subscapularis pathology.

Finally, the relations between acromiohumeral distance and the results of the clinical impingement signs were examined. The mean (SD) acromiohumeral distance of subjects with MRI evidence of current or previous supraspinatus

\begin{tabular}{|c|c|c|}
\hline Supraspinatus pathology & Spur present & Spur absent \\
\hline $\begin{array}{l}\text { Supraspinatus tear/tendonitis } \\
\text { Supraspinatus normal }\end{array}$ & $\begin{array}{l}6 \\
5\end{array}$ & $\begin{array}{l}4 \\
37\end{array}$ \\
\hline $\mathrm{n}=52 ; \mathrm{p}=0.003$ (Fisher's & kact test). & \\
\hline
\end{tabular}

pathology was $0.32(0.09) \mathrm{cm}$ and $0.35(0.05) \mathrm{cm}$ in subjects with no evidence of supraspinatus pathology $(p=0.61)$. The mean acromiohumeral distance of subjects in whom the Centinela test or the resisted external rotation test was positive was not significantly reduced $(\mathrm{p}=0.144$ and $\mathrm{p}=$ 0.641 respectively).

\section{DISCUSSION}

In accordance with the study of Cox and Nouwen on sprint kayakers, cited in Edwards, ${ }^{6}$ soft tissue injuries of the rotator cuff make up a large portion of the injuries seen in our study. Pathology of the supraspinatus muscle/tendon was seen in $19 \%$ and of the subscapularis muscle/tendon in $3.8 \%$ of our 52 subjects, about half of whom had been symptomatic or had abnormalities on MRI. This number of rotator cuff abnormalities is about twice the number reported by Cox and Nouwen. The discrepancy is not surprising when the differences between sprinting and marathon kayaking are considered and, probably more importantly, the difference in the results between physical examination and MRI.

Kayaker's age, number of years kayaking, and number of endurance races completed do not relate significantly to symptoms or abnormalities on MRI. Age, number of years kayaking, and number of races completed, when related to the presence or absence of acromioclavicular hypertrophy, show no significant correlation whatever. This suggests that its presence in many cases may be the result of portaging or an acute incident, such as a previous injury, and not due to overuse.

Pathoanatomical features that predispose to rotator cuff injury in kayakers were searched for by correlating symptoms, both current and previous, with the presence or absence of downsloping of the acromion, acromioclavicular hypertrophy, and os acromiale, but no significant relations were evident. This finding is partly in contrast with the report of Hawkins and Kennedy, ${ }^{11}$ which describes a significant relation between acromioclavicular hypertrophy and rotator cuff impingement.

Acromioclavicular spurs, on the other hand, correlate highly with supraspinatus muscle pathology, found in $54.5 \%$ of shoulders in which a spur was evident on MRI. This percentage is lower than found by Bigliani et al, who showed that $70 \%$ of shoulders with subacromial spurs were associated with tears of the rotator cuff.

Many authors, particularly proponents of Neer's hypothesis, have reported a significant relation between decreased subacromial space and the development of rotator cuff impingement. No such relation was shown in our study. Both acromiohumeral distance and coracohumeral distance failed to correlate with the presence of shoulder symptoms. Similarly, rotator cuff muscle hypertrophy has been claimed to increase the incidence of rotator cuff pathology, ${ }^{23}$ but no significant relation could be shown when supraspinatus and subscapularis diameter was compared with the presence of symptoms, not even when muscle diameter and bony distance were expressed as a ratio for each subject.

The above findings suggest that at least a large part of the rotator cuff pathology identified in the shoulders of these kayakers is due to factors other than subacromial impingement. Rather, impingement appears to be exclusively related to the presence of acromioclavicular spurs and not to pathoanatomical changes in the coracoacromial arch or rotator cuff musculature. Therefore this study appears to bear out the idea that primary impingement plays little or no role at all in kayakers, particularly if it is assumed that the spurs are the result, and not the cause, of the degenerative changes observed in the shoulders.

In conclusion, the results of our study of marathon kayakers suggest that primary impingement of the rotator 
cuff, as identified in this group of subjects, is not due to spatial restrictions around the shoulder joint. Rather, the observed rotator cuff pathology is the result of secondary impingement factors associated with overuse and is possibly specific to kayakers. Direct, primary-type impingement may still occur during the kayak stroke when the pushing shoulder forces the greater tuberosity of the humerus against the anteroinferior edge of the acromion, thereby impinging on the supraspinatus muscle/tendon. It is impossible to assess the contribution of each of these factors.

In addition, as stated above, the cause of the acromioclavicular spurs and their role in impingement remains far from clear.

It is important to emphasise that the above findings and conclusions pertain to marathon kayakers only and not to kayakers in general. The severe selection criteria for participation in this study bear out this point. It is quite possible that bony restrictions around the shoulder, eventually leading to primary impingement, play as significant a role among kayakers as they play among the population at large, but were absent in our study group on account of the selection process. Any anatomical feature that may potentially lead to primary impingement may have forced some kayakers to give up the sport prematurely thereby excluding themselves from eligibility.

Another case in point may be the absence of supraspinatus and subscapularis muscle hypertrophy, a feature that has been well documented as a cause of impingement seen in weightlifters and paddlers. ${ }^{23}$ Whereas one would intuitively expect this type of muscle hypertrophy to be common among marathon kayakers, its absence in our study group may indicate once again that kayakers suffering from this potential cause of impingement are not represented or are underrepresented in this self selected sample of subjects.

On the other hand, because subjects were not selected randomly, but volunteered of their own accord, certain shoulder injuries may have been overrepresented in this group, as kayakers with painful symptoms may have been more inclined to participate in this study than those who were pain-free. Despite the selection process, a reasonable spread of subjects with regard to the presence or absence of symptoms was obtained, with about half of the kayakers asymptomatic. At least $83 \%$ of the symptomatic subjects had chronic overuse injuries in that they could not remember any single incident causing their shoulder symptoms. This incidence is likely to be higher than in the global kayaking population because of the gruelling nature of marathon kayaking.

In summary, overuse injuries to the shoulder of marathon kayakers are quite common, with rotator cuff abnormalities about twice as common as seen in sprint kayakers. Age, number of years kayaking, and number of races completed do not correlate significantly with pathoanatomical features identified by physical examination or MRI. Only acromioclavicular spurs appear to correlate highly with supraspinatus muscle pathology, but it remains uncertain if these are the result, or the cause, of the degenerative changes observed in the shoulder.

\section{Authors' affiliations \\ G Hagemann, M Mars, University of Natal, Durban, South Africa \\ A M Rijke, University of Virginia}

\section{REFERENCES}

1 Burrell CL, Burrell R. Injuries in white-water paddling. Phys Sportsmed 1982;10:119-24.

2 Ganthier MM. Preventing injuries among watercraft padlers: steady as you go! Phys Sportsmed 1989; 17:163-9.

3 Murphy R. Ultra sports in spite of injuries. Phys Sportsmed 1986;14:180-9.

4 Kizer KW. Medical aspects of white-water kayaking. Phys Sportsmed 1987; 15:128-37.

5 Stepkovitch N, Stepkovitch W, Hawkins P, et al. Proceedings of the 25th anniversary bicentennial conference. Sydney: Australian Sports Medicine Federation Ltd, 1988:19-28.

6 Edwards A. Injuries in kayaking. Sport Health 1993;11:8-11.

7 Walsh M. Sports medicine for paddlers: the cause, care and treatment of paddlers injuries. CANOE 1989;17:36-8.

8 Zuluaga M, Briggs C, Carlisle J, et al. Sports physiotherapy: applied science and practice. Melbourne: Churchill Livingstone, 1995.

9 Neer CS. Anterior acromioplasty for the chronic impingement syndrome in the shoulder: a preliminary report. J Bone Joint Surg [Am] 1972;54:41-50.

10 Neer CS. Impingement lesions. Clin Orthop 1983;173:70.

11 Hawkins RJ, Kennedy JC. Impingement syndrome in athletes. Am J Sports Med 1980;8:151-7

12 Nirschl RP. Prevention and treatment of elbow and shoulder injuries in the tennis player. Clin Sports Med 1988;7:289-308.

13 Nirschl RP. Rotator cuff tendinitis: basic concepts of pathoethiology. Instructions Course Lecture of AAOS 1989:38.

14 Fowler HF. Shoulder injuries in the mature athlete. Advances in Sports Medicine and Fitness 1988;1:225-38.

15 Perry J. Anatomy and biomechanics of the shoulder in throwing, swimming, gymnastics and tennis. Clin Sports Med 1983;2:247-70.

16 Schenkman M, Rugo de Caraya V. Kinesiology of the shoulder complex. Journal of Sports Physical Therapy 1987;8:438-50.

17 Falkel J, Murphy T. Shoulder injuries: sports injury management. Baltimore: Williams and Wilkins, 1988.

18 Ferrari D. Capsular ligaments of the shoulder. Anatomical and functional study of the anterior capsule. Am J Sports Med 1990;18:20-4.

19 Blackburn T. Throwing injuries to the shoulder: physical therapy to the shoulder. New York: Churchill Livingstone, 1987.

20 Jobe FW, Moyns D. Delineation of diagnostic criteria and a rehabilitation program for rotator cuff injuries. Am J Sports Med 1982;10:336-9.

21 Yocum L. Assessing the shoulder. Clin Sports Med 1983;2:281-9.

22 Bigliani LU, Morrison DS, April EW. The morphology of the acromion and its relationship to rotator cuff tears. Orthopaedic Transactions 1986;10:228.

23 Pelham TW, Holt LE, Stalker RE. The etiology of paddlers shoulder. Aust J Sci Med Sport 1995;27:43-7. 